\title{
Constructing Neural Networks by Extending the Optimization Field
}

\author{
Wenyuan Zhang \\ Huazhong University of Science and Technology \\ u201613687@hust.edu.cn
}

\begin{abstract}
The performance of the neural network is not unilaterally increased with the training parameters of the neural network, and it is possible that the parameters of the training ground become more and the performance is degraded. This paper explains what the optimization field is and proposes a new way to solve this degradation problem. The new method is to built a neural network which includes the previous optimization field while adding parameters and extends the new optimization field .From this point, the paper explains the excellence of Inception, ResNet and DenseNet networks. Furthermore, this paper also proposes a new method for constructing neural networks that can improve the performance of neural networks with the increase of training parameters.
\end{abstract}

\section{CCS Concepts}

- Computing methodologies $\rightarrow$ Neural networks • Computing methodologies $\rightarrow$ Supervised learning

\section{Keywords}

Degradation problem, Inception, ResNet, DenseNet, new method to construct network

\section{INTRODUCTION}

The improvement of computer computing power has laid the foundation for the prosperity of neural networks. With the increase of computer computing speed, the emergence of GPU and TPU, the development trend of neural network is that the training parameters are increased and the training depth is increased such as the VGG network ${ }^{[1]}$ and the LeNet network $^{[2]}$. This way, the neural network's property has been greatly improved since the robust property of VGG network and LeNet network has been proven in various tasks ${ }^{[2,3,4,5]}$. However, it has recently been found that the performance of neural networks is not a single positive relationship with the increase of the number of layers and the increase of training parameters. The performance of the neural network deteriorates as the number of layers increases, but this is not caused by underfitting or overfitting. This phenomenon indicates that the neural network is so complex that after reaching a certain level, using the above two

Permission to make digital or hard copies of all or part of this work for personal or classroom use is granted without fee provided that copies are not made or distributed for profit or commercial advantage and that copies bear this notice and the full citation on the first page. Copyrights for components of this work owned by others than ACM must be honored. Abstracting with credit is permitted. To copy otherwise, or republish, to post on servers or to redistribute to lists, requires prior specific permission and/or a fee. Request permissions from Permissions@acm.org.

ESSE 2020, November 6-8, 2020, Rome, Italy

(C) 2020 Association for Computing Machinery.

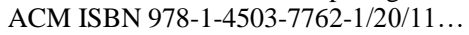

https://doi.org/10.1145/3393822.3432313 methods to use computer computing power as much as possible may not continue to improve the performance of the neural network. Based on this, this paper proposes a method that can train the neural network using computer computing power as much as possible while ensuring that the performance of the neural network does not decrease.

Before explaining this method, let us do such a thought experiment as follows. If there already have a versatile computer and you can optimize your neural network to the best, what method we should take to design a neural network? Is it feasible to follow the current practice to increase the parameters of neural network training? We record all the parameters of the training as $W$, the input of the neural network is recorded as $X$, the output is recorded as $Y$, and the forward propagation of the neural network can be represented by a function $Y=f(W, X)$, we optimize the best parameters Recorded as $W^{\prime}$, the best function for optimization is $Y=f\left(W^{\prime}, X\right)$. Now we have two networks with different numbers of parameters, the parameters are, $W_{1}$ and $W_{2}$ respectively. The final results of the two network optimizations are $Y=f\left(W_{1}^{\prime}, X\right)$ and $Y=f\left(W_{2}^{\prime}, X\right)$. Now we need to solve such a problem, whether network 2 optimized to the best performance must be better than network 1 , or whether we can find a third network which has the performance after optimization is not worse than that of network 1 and network 2 optimized to their best. Now we do such a thing, because the number of parameters of the training is different, then the hyperparameters of the networks must be different. If we increase the parameters by increasing the number of layers of the neural network, then the different hyperparameters are the number of layers in the network. If we increase the parameters by changing the size of the cnn filter, then the different hyperparameters are the size of the filter. We now record the hyperparameters of this change as $S, S$ in network 1 and network 2 are respectively $S_{1}$ and $S_{2}$ Then $S_{1}$ and $S_{2}$ are added to the forward-propagating function. At this time, the two final functions of the optimization are $Y=f\left(S_{1}, W_{1}^{\prime}, X\right)$ and $Y=f\left(S_{2}, W_{2}^{\prime}, X\right)$. Because the hyperparameters in the training process are not involved in training, they are fixed, but this brings a problem. Because the network have different hyperparameters, it can be said that the two networks are optimizing their performance in different regions. We refer these region as optimization field.

We now design a hyperplane as shown in Figure 1. The abscissa is the parameter $\mathrm{W}$ and the ordinate is the hyperparameter S. It can be seen from the figure that the neural network 1 and the 
neural network 2 actually find the optimal solution on two different straight lines, respectively. These two lines do not intersect and it is very difficult to compare which solution area is superior to the other one. So we came up with such a solution, we do not have to restrict us to solve the optimal solution in one of the regions, we can solve the optimal solution in these two regions or even more regions. The result of the final optimization of this approach must not be worse than solving the problem only in one solution region, because the global optimal solution with a wider optimization field must be better than the local optimal solution whose optimization area is contained in the area of global optimal solution.

Our previous practice was to change a hyperparameter $\mathrm{S}$, which added more trainable variables. But this method just replaces a solution area and finds the optimal solution in this area, and the result may be worse than before. But the way we can do now is to solve it in a larger and wider area. This area contains the previous area, and it has the better performance than the previous network.

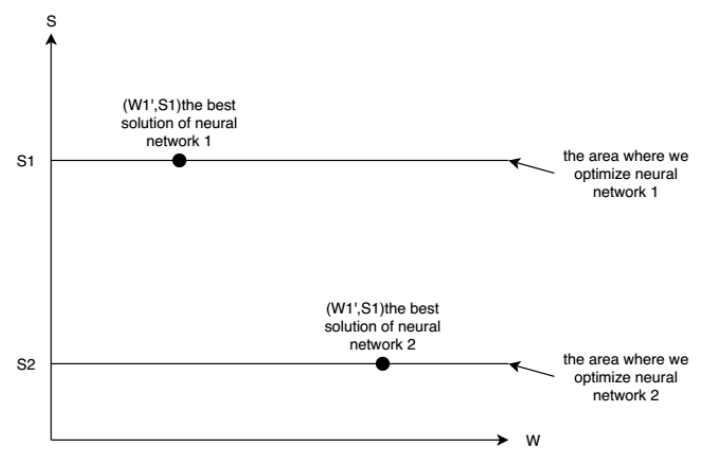

Figure 1. The figure above presents the optimization area of neural network 1 and neural network 2 and mark the point of the best solutions of each neural network.

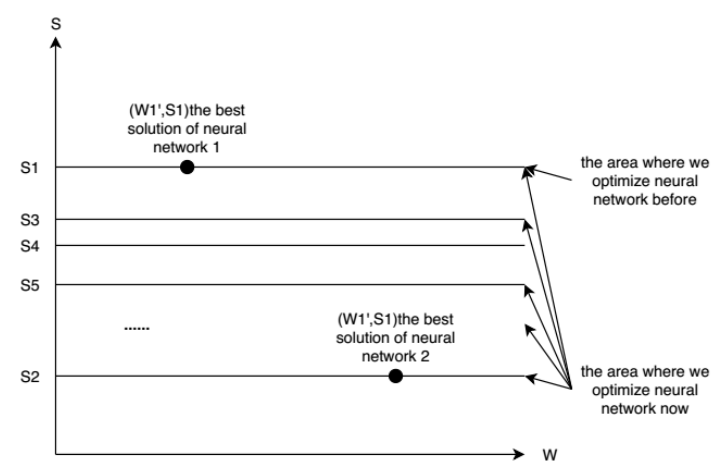

Figure 2. The figure above presents the areas where we optimize neural network before and where we optimize neural network now.

It is not easy to understand the solution area represented by the hyperplane coordinate system. Because the line does not represent a block, the $\mathrm{S}$ in the figure cannot be continuously changed, so we use the Wayne diagram for a more intuitive understanding. Depending on the hyperparameters selected, different hyperparameters may have an intersection, meaning that mixed hyperparameters can be used. Our previous optimization area and the optimization area to be expanded now are shown in Figure 3.

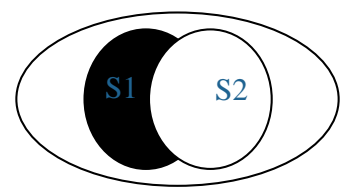

the area where we optimize before

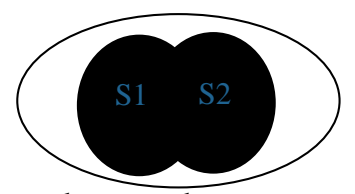

the area where we optimize now

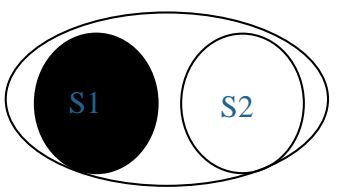

the area where we optimize before

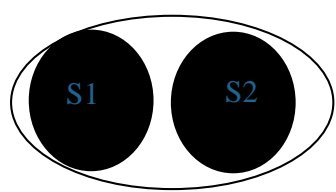

the area where we optimize now
Figure 3. The figure above depicts the optimization area by using the Wayne diagram.

\section{EXTENDED OPTIMIZATION FIELD NEURAL NETWORKS \\ 2.1 Inception ${ }^{[6]}$}

Due to the large differences in information locations, it is more difficult to select the appropriate convolution kernel size for the convolution operation. If the information distribution is more global, the image prefers a larger convolution kernel, and the information distribution is more local to the convolution kernel with smaller image preference. So how to choose the appropriate convolution kernel size this hyperparameter becomes a problem. A $1 * 1$ convolution kernel should be used more near the input layer, and $3 * 3$ and $5 * 5$ convolution kernels should be used near the output ${ }^{[6]}$, but it is not clear that which is the layer that we should use $1 * 1$ convolution kernels before and we should use $3 * 3$ and $5 * 5$ convolution kernels after. The method of clearly determining which filters to use in each layer is through a constant adjustment, which must be a heavy job. But the Inception network provides another method.

Inception's initial layer of modules is shown in Figure 4. As can be seen from the figure, the input-to-output of one layer does not pass through a single convolution kernel, but passes through three convolution kernels. This approach potentially extends the area of parameter optimization. This approach is undoubtedly better than just a single convolution kernel, because for a network that only passes through a single convolution kernel equals to the Inception unit whose weights in the other two convolution kernels are all zero. Therefore, the region optimized by the single convolution kernel is included in the area optimized by the Inception network, and this optimization field is a special part of the Inception unit where two filters of the neural network have the weights which all equal to zero. Therefore, if the optimization ends up with only one filter, the inception network can certainly be optimized to the position where the weights of the other two convolution kernels are all zero. So the inception network is a network which is superior to the network which uses only a single convolution kernel. And the robust performance of Inception has been proven $^{[6]}$

But more importantly, the inception network is not just a combination of optimized regions that use only a single convolution kernel. We can see that it also adds many other optimization regions. 


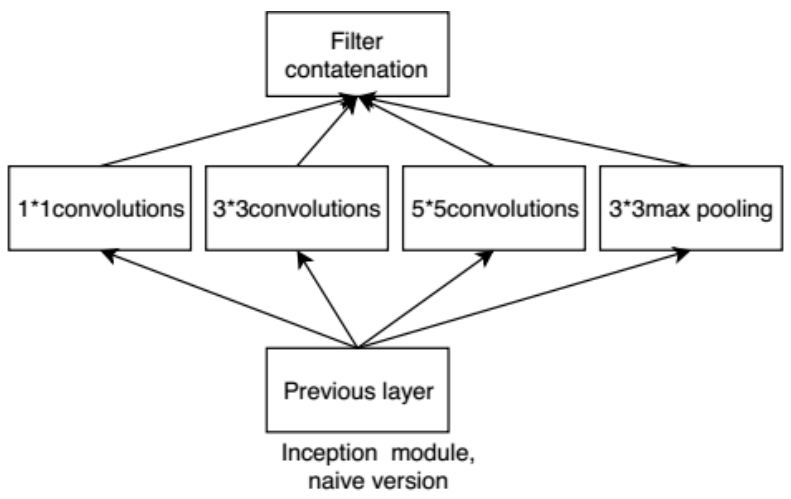

Figure 4. The figure above shows the inception units.

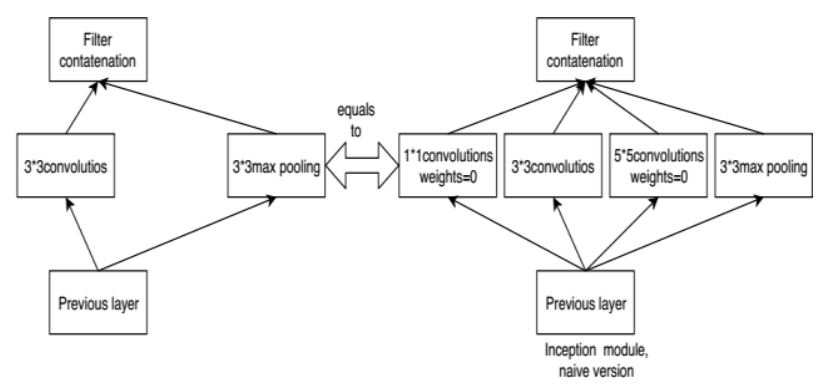

Figure 5. The figure above demonstrates that a network that only passes through a single convolution kernel equals to the Inception unit whose weights in the other two convolution kernels are all zero.

Figure 6 shows the optimization domain with a single convolution kernel and the optimization domain using the inception network in the hyperplane coordinate system. It can be seen that after we use the inception network, the neural network optimization area is not only the union of the three convolution kernel regions used previously, but also adds four regions, that is, at least two convolution kernels are used at the same time. An example is shown in Figure 7.

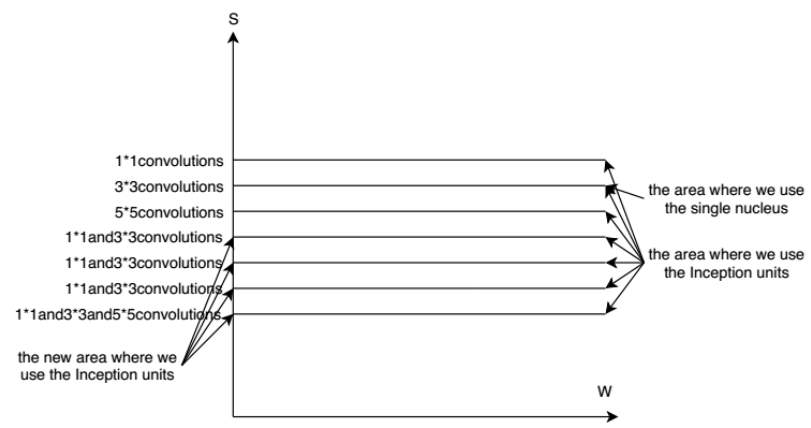

Figure 6. The figure above shows the optimization area where we use the Inception unit.

\section{2 $\operatorname{ResNet}^{[7]}$}

The performance of the neural network does not increase correspondingly as the number of layers increases. As the depth of the neural network increases, the accuracy reaches saturation and degrades rapidly, but unexpectedly, this degradation is not caused by underfitting or overfitting, and adding more layers in a reasonable neural network model leads to more High error rate ${ }^{[7]}$.
This phenomenon is also proved in the experiment, as shown in Figure 8.

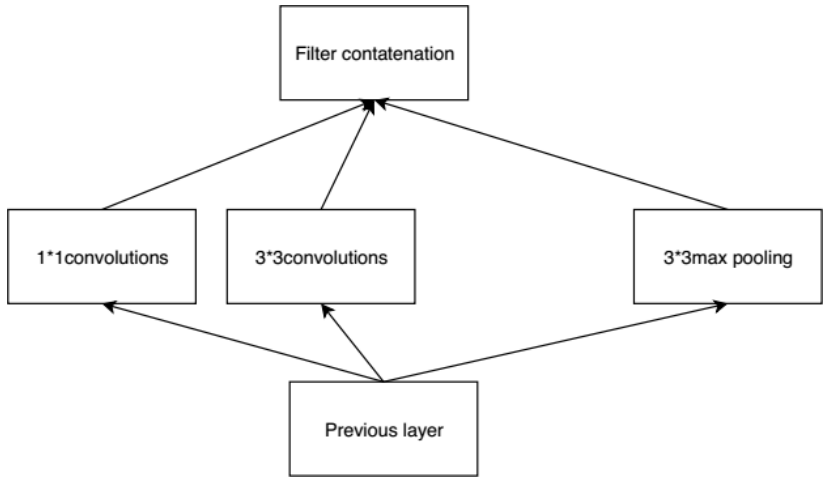

Figure 7. The figure above gives an example that at least two kernels are used in the Inception unit.
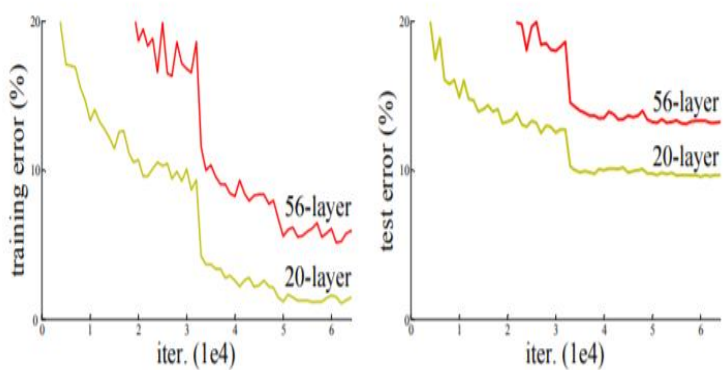

Figure 8. (source:[7]) The figure above illustrates the phenomenon that as the depth of the neural network increases, the accuracy reaches saturation and degrades.

The solution presented in an article ${ }^{[7]}$ is: For deeper models, use the identity map to build the newly added layer, while the other layers are the same as the previous shallow model. Later, this article again proposed the ResNet unit, as shown in Figure 9. The article explains why the ResNet network can solve the degradation problem: if the depth of the neural network is optimized to the beat when it reaches a certain layer, then the residual map of some ResNet units will become 0, so those ResNet units are equivalent to only after doing an identity mapping, those layers of the ResNet unit are equivalent to nothing. Therefore, although the depth of the ResNet network is deeper than the optimal depth network, the optimization is finally equivalent to the optimal depth network. This method is quiet effective since ResNet had been proven strong in various

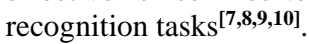

Using the ideas presented in this paper, we can explain this problem by adding a ResNet unit that expands the optimization field and always contains the original optimization domain because if the weight of the branch of the residual is all zero, it is equivalent to the neural network that does not increase the ResNet unit layer, and the optimization field of the neural network is reduced to the original optimization field as shown in Figure 10 . Therefore, while continuously increasing the number of network layers with the ResNet unit, the optimization field of the neural network will continue to expand while including the optimized domain. Therefore, when the number of neural network layers is increased by the ResNet unit, the performance of the neural 
network will always follow the number of layers- increased by the increase.

The optimization field of the neural network of the Resnet is represented in the hyperplane as shown in Figure 11.

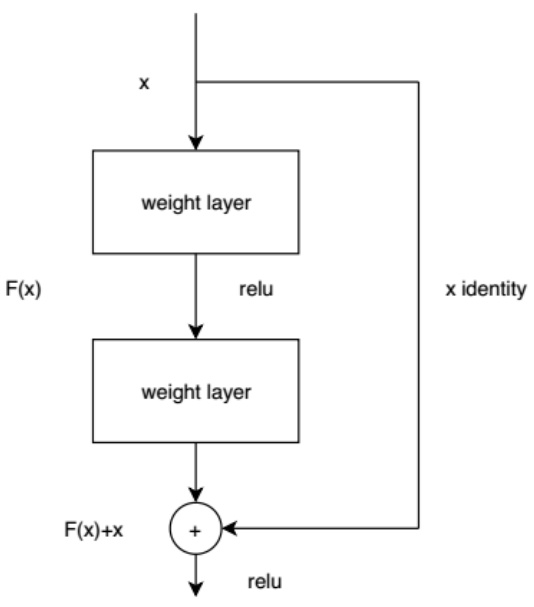

Figure 9. The figure above shows a ResNet unit.

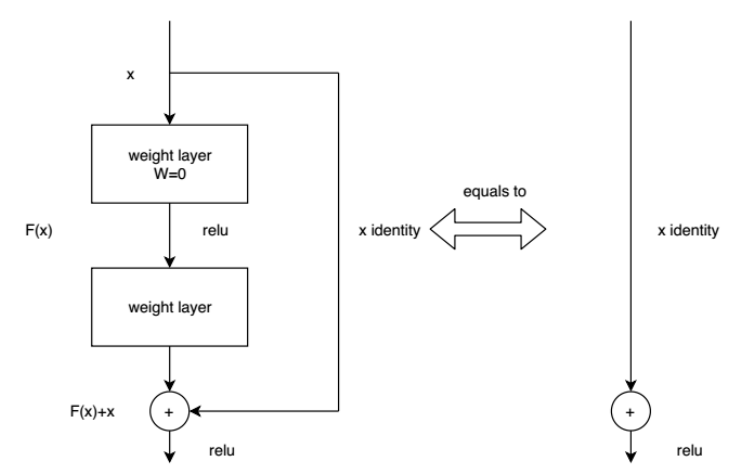

Figure 10. The figure above demonstrates that if the weight of the branch of the residual is all zero, it is equivalent to the neural network that does not increase the ResNet unit layer.

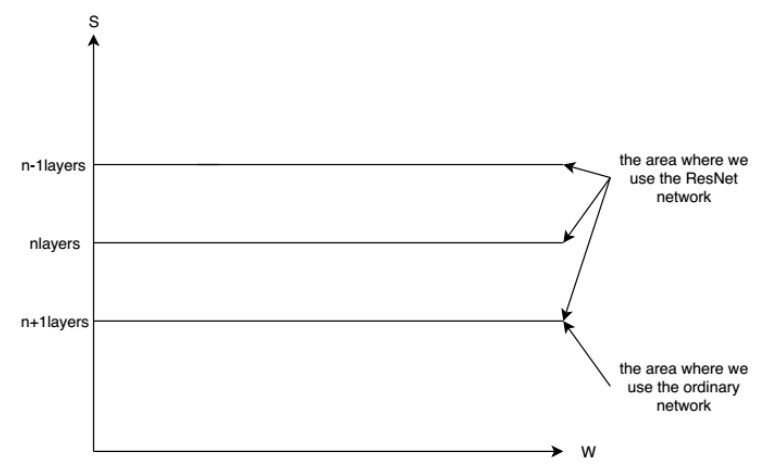

Figure 11. The figure above depicts the optimization fields where we use the ResNet neural network and the original neural networks.

\subsection{Densenet $^{[10]}$}

A unit structure of the DenseNet network is shown in figure 12. In a general neural network, each layer is only connected to the next deeper layer, but the DenseNet network layers are connected to all the deeper layers ${ }^{[3]}$.

The same thing about the DenseNet network and the Resnet network is that as the number of neural network layers increases, the neural network optimization domain expands while still including the previous optimization field, because we only need to make the convolution weight of a certain layer all zero. Without this layer, as shown in figure 13, the optimization domain of the neural network is reduced to an optimization field that is one layer less than the original network.

However, in addition to retaining the optimization field of different layers, DenseNet has a wider optimization field than Resnet, because the optimization field may be different even if the amount of layers is the same. For example, the same two-layer network, as long as the selection of layers is different, the optimized area is also different. The two-layer network of different optimized areas is shown in figure 14, and the optimized area of the two-layer network is shown in figure 15. As a result, the DenseNet network performs better than Resnet's network, which also confirms the opinion that from the receptive field, the shallow and deep fields can be combined more freely, which will make the model has a better performance ${ }^{[10]}$.

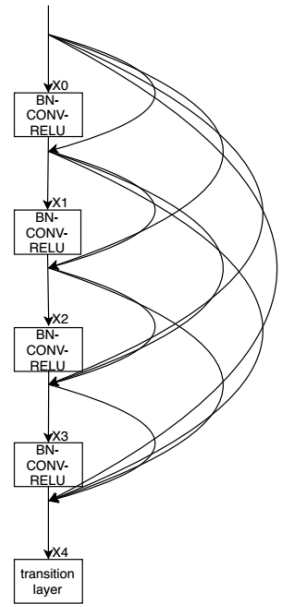

Figure 12. The figure above shows a block of DenseNet.

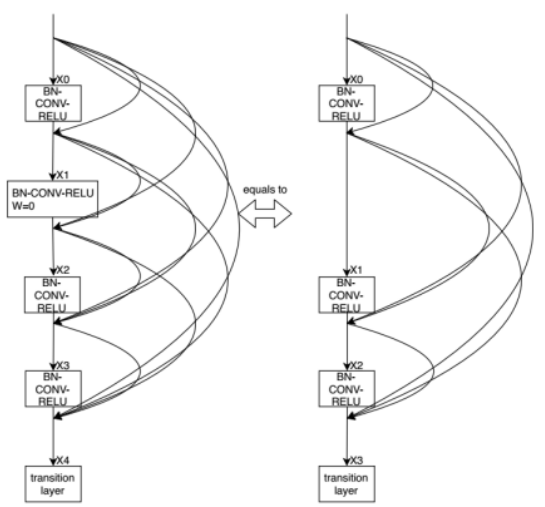

Figure 13. The figure above shows that the optimization field of the neural network is reduced to an optimization field that is one layer less than the original network 


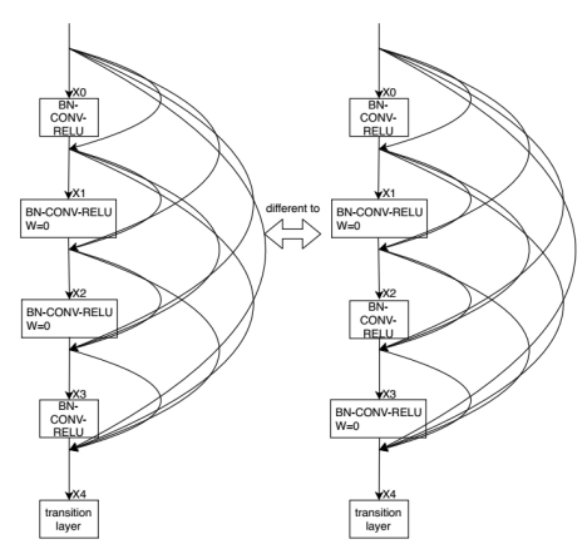

Figure 14. The figure above shows the two-layer networks which have different optimization fields.

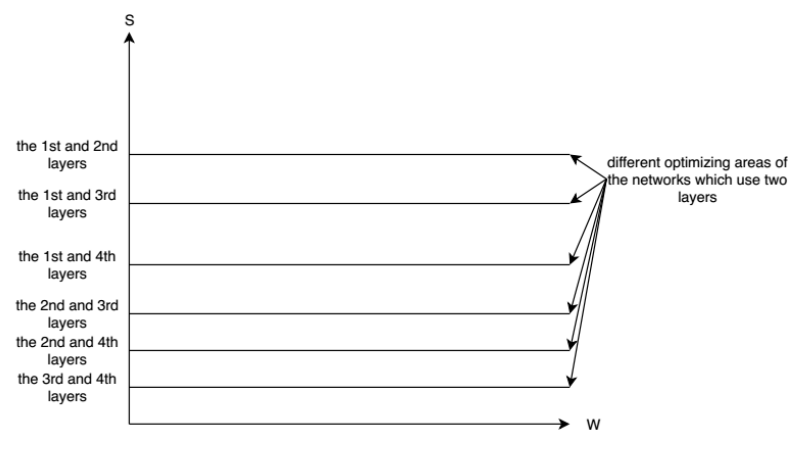

Figure 15. The figure above shows the two-layer networks which have different optimization fields.

\section{METHOD OF CONSTRUCTING NEURAL NETWORK}

With computer computing power developing fast, the depth of the neural network has gradually deepened, and the parameters of training have gradually increased. However, if you only increase the amount of optimization parameters, regardless of whether the network changes its optimization field and not covers the previous area, this approach may have little effect, just simply changing the optimization field, greatly wasting computing resources. Therefore, our future network design idea must be to modify the network at the same time to cover the previous optimization domain, which is to truly expand the optimization field, so that the network performance has been really improved, no waste of computing resources. The idea of our transformation is shown in Figure 16. In the figure, the optimization field points to a network that indicates which optimization field belongs to that network.

\section{CONCLUSIONS}

The problem of degradation of the neural network is caused by not considering the optimization field. If a neural network includes the previous optimization field while adding parameters and extends the new optimization field, then degradation will not occur if the computer resources are sufficient. Inception, ResNet and DenseNet are built in different ways to extend the idea of enlarging the optimization field, so they all have excellent properties. Finally, we must follow the principle of expanding the optimization field when designing a new neural network.

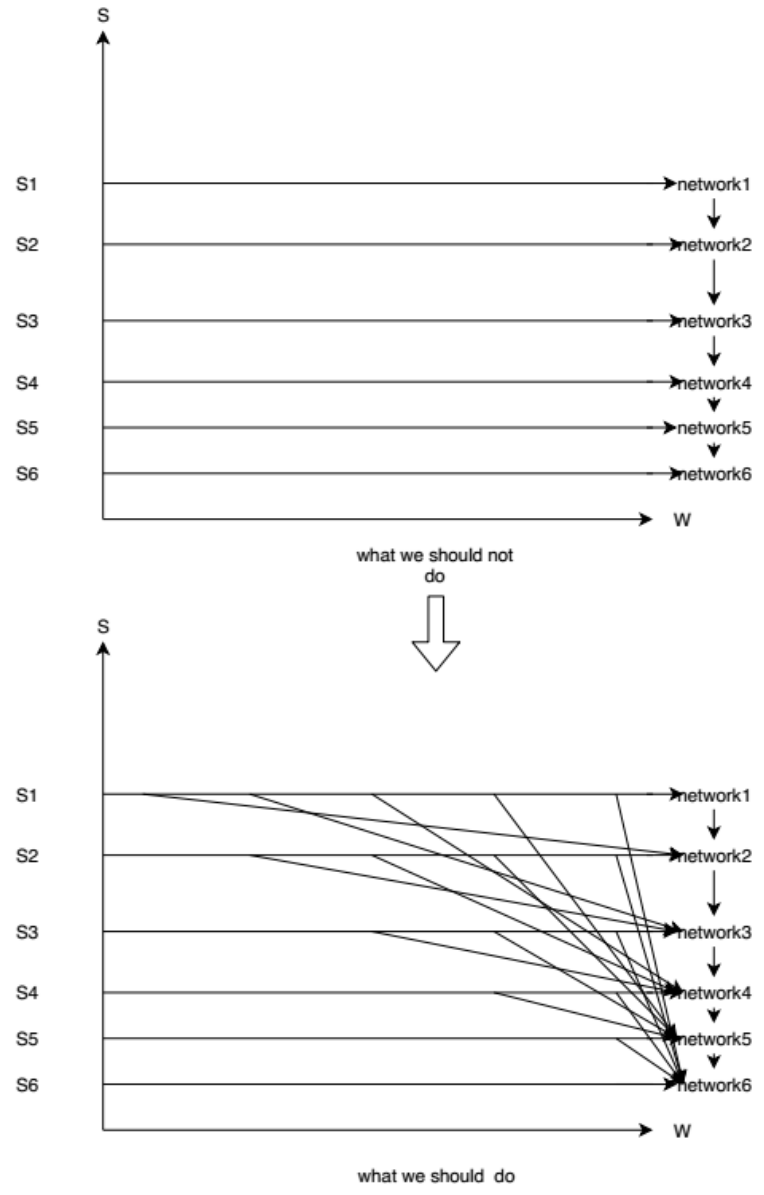

Figure 16. The figure above illustrates how to develop a neural network.

\section{REFERENCES}

[1] Simonyan K, Zisserman A. Very deep convolutional networks for large-scale image recognition[J]. arXiv preprint arXiv:1409.1556, 2014.

[2]. LeCun Y, Bottou L, Bengio Y, et al. Gradient-based learning applied to document recognition[J]. Proceedings of the IEEE, 1998, 86(11): 2278-2324.

[3] J. Donahue, Y. Jia, O. Vinyals, J. Hoffman, N. Zhang, E.Tzeng,andT.Darrell. Decaf: Adeepconvolutional activation feature for generic visual recognition. In ICML, 2014.

[4] R.Girshick,J.Donahue,T.Darrell,andJ.Malik. Rich feature hierarchies for accurate object detection and semantic segmentation. In CVPR, 2014.

[5] R. Girshick. Fast R-CNN. In ICCV, 2015.

[6] Szegedy C, Liu W, Jia Y, et al. Going deeper with convolutions $[\mathrm{C}] / /$ Proceedings of the IEEE conference on computer vision and pattern recognition. 2015: 1-9.

[7] He K, Zhang X, Ren S, et al. Resnet-Deep Residual Learning for Image Recognition[J]. ResNet: Deep Residual Learning for Image Recognition, 2015.

[8] J. Long, E. Shelhamer, and T. Darrell. Fully convolutional networks for semantic segmentation. In CVPR, 2015. 
[9] Pinheiro P O, Collobert R, Dollár P. Learning to segment object candidates[C]//Advances in Neural Information Processing Systems. 2015: 1990-1998.

[10] Huang G, Liu Z, Van Der Maaten L, et al. Densely connected convolutional networks[C]//Proceedings of the IEEE conference on computer vision and pattern recognition. 2017: $4700-4708$. 